Bull. Austral. Math. Soc.

Vol. 42 (1990) [253-266]

\title{
APPROXIMATION BY BOOLEAN SUMS OF LINEAR OPERATORS: TELYAKOVSKII-TYPE ESTIMATES
}

\author{
Jia-Ding CaO and Heinz H. Gonska
}

In the present note we study the question: "Under which general conditions do certain Boolean sums of linear operators satisfy Telyakovskir-type estimates?" It is shown, in particular, that any sequence of linear algebraic polynomial operators satisfying a Timan-type inequality can be modified appropriately so as to obtain the corresponding upper bound of the Telyakovskif-type. Several examples are included.

\section{INTRODUCTION}

Let $N=\{1,2, \ldots\}$ be the set of natural numbers. For $f \in C[a, b]$ (real-valued and continuous functions on the compact interval $[a, b])$, let $\|f\|:=\max \{|f(t)|: a \leqslant$ $t \leqslant b\}$ denote the Cebyšev norm of $f$. By $c, \tilde{c}$ we denote positive absolute constants independent of $n, f$, and $x \in[a, b]$. The constants $c$ and $\tilde{c}$ may be different at different occurrences, even on the same line. Let $\Pi_{n}$ be the set of algebraic polynomials of degree $\leqslant n$. For $f \in C[a, b]$, the modulus of continuity of $f$ is defined by

$$
\omega(f, \delta):=\sup \left\{\left|f\left(x_{1}\right)-f\left(x_{2}\right)\right|:\left|x_{1}-x_{2}\right| \leqslant \delta\right\}, \quad 0 \leqslant \delta \leqslant b-a .
$$

In his well-known paper [26] Timan (see also [27]) proved the following

THEOREM A. For $n \in N$ and $f \in C[-1,1]$ there exists $P_{n}(f, \cdot) \in \Pi_{n}$ such that

$$
\left|f(x)-P_{n}(f, x)\right| \leqslant c \cdot \omega\left(f,\left(1-x^{2}\right)^{1 / 2} \cdot n^{-1}+n^{-2}\right),|x| \leqslant 1 .
$$

Telyakovskii [25] improved this to

ThEOREM B. For $n \in N$ and $f \in C[-1,1]$ there exists $P_{n}(f, \cdot) \in \Pi_{n}$ such that

$$
\left|f(x)-P_{n}(f, x)\right| \leqslant c \cdot \omega\left(f,\left(1-x^{2}\right)^{1 / 2} \cdot n^{-1}\right),|x| \leqslant 1 .
$$

Theorem B provided a partial answer (that is, for the case of arbitrary continuous functions) to a problem posed by Lorentz $[17$, p.185] in 1963 . Quite elementary proofs

Received 26 October 1989

Copyright Clearance Centre, Inc. Serial-fee code: 0004-9729/90 SA2.00+0.00. 
of Theorems A and B were given by Pičugov and Lehnhoff (see $[21,14,15]$ ) who used operators of the following type.

Let, for $f \in C[-1,1]$ and $n \in N$,

$$
\begin{aligned}
K_{m(n)}(v) & :=\frac{1}{2}+\sum_{k=1}^{m(n)} \rho_{k, m(n)} \cos k v, \text { and } \\
G_{m(n)}(f ; x) & :=\frac{1}{\pi} \int_{-\pi}^{\pi} f(\cos (\arccos x+v)) \cdot K_{m(n)}(v) d v .
\end{aligned}
$$

Here, the kernel $K_{m(n)}$ is a trigonometric polynomial of degree $m(n)$ such that

(i) $K_{m(n)}$ is positive and even, and

(ii) $\int_{-\pi}^{\pi} K_{m(n)}(v) d v=\pi$.

This implies that $G_{m(n)}(f, \cdot)$ is an algebraic polynomial of degree $m(n)$. For $s \in \mathrm{N}$, the Masuoka (higher order Jackson) kernels are given by

$$
K_{e n-\infty}(v)=c_{n, \infty}\left[\frac{\sin (n v / 2)}{\sin (v / 2)}\right]^{2,},
$$

where $c_{n, \mathbb{e}}$ is chosen such that $\pi^{-1} \int_{-\pi}^{\pi} K_{e_{n-1}}(v) d v=1$.

In his second paper Lehnhoff investigated certain Boolean sum modifications of the operators $G_{m(n)}$ in order to prove Theorem B. His research was continued by the second author in $[10,11]$, and by the first in $[1,2]$. Both investigated Timan-type estimates for the $G_{m(n)}$ and Telyakovskii-type estimates for their modifications $G_{m(n)}^{+}$. In [3] the present authors investigated approximation by Boolean sums of positive linear operators.

In this article, we study the more general problem of approximation by Boolean sums of linear operators. We establish several general results. Our central Theorem 3 shows that, from a Timan-type estimate for linear algebraic polynomial operators $A_{n}$, one can always derive a Telyakovskii-type estimate for their Boolean sum modifcations $A_{n}^{+}$. This fact is applied to certain operators $G_{m(n)}$ having the property that $1-\rho_{1, m(n)}=\mathcal{O}\left(n^{-2}\right), n \rightarrow \infty$. Furthermore, in Section 4 we give applications to Bernstein-Schurer polynomials, Kantorovič polynomials, and Durrmeyer polynomials.

\section{TWO GENERAL THEOREMS}

Let $f \in C[a, b]$ and $L f$ denote the linear function interpolating $f$ at $a$ and $b$, that is,

$$
L(f, x):=\frac{f(b)(x-a)+f(a)(b-x)}{b-a}, \quad a \leqslant x \leqslant b .
$$


Let $A: C[a, b] \rightarrow C[a, b]$ be a linear operator. Then the Boolean sum $L \oplus A$ of $L$ and $A$ is given by

$$
A^{+}:=L \oplus A=L+A-L \circ A
$$

More explicitly,

$$
A^{+}(f, x)=A(f, x)+(b-a)^{-1}\{(x-a)[f(b)-A(f, b)]+(b-x)[f(a)-A(f, a)]\}
$$

Note that, if $A(f, a)=f(a)$ and $A(f, b)=f(b)$ for all $f \in C[a, b]$, then $A^{+}=A$.

For $k \in \mathrm{N} \cup\{0\}$, let $C^{k}[a, b]$ denote the space of $k$-fold continuously differentiable functions. Hence $f \in C^{k}[a, b]$ means that $f^{(k)} \in C[a, b]$. Using the $K$-functional technique, one obtains the following:

Lemma 1. (see, for example, DeVore [7]). Let $H_{n}: C[a, b] \rightarrow C[a, b]$ be a sequence of linear operators, satisfying the following conditions:

(i) $\left\|H_{n} f\right\| \leqslant c \cdot\|f\|$ for all $f \in C[a, b]$.

(ii) For $a \leqslant x \leqslant b, 0 \leqslant \varepsilon_{n}(x) \leqslant b-a$ and $h \in C^{1}[a, b]$, one has $\left|H_{n}(h, x)-h(x)\right| \leqslant c \cdot \varepsilon_{n}(x) \cdot\left\|h^{\prime}\right\|$.

Then for all $f \in C[a, b],\left|H_{n}(f, x)-f(x)\right| \leqslant c \cdot \omega\left(f, \varepsilon_{n}(x)\right)$.

In the following theorem it is shown that Telyakovskii-type estimates for operators of the $L \oplus A$ type hold under quite general conditions.

ThEOREM 1. Let $A_{n}: C[a, b] \rightarrow C^{1}[a, b]$ be a sequence of linear operators, satisfying the following conditions:

(i) $\left\|A_{n} f\right\| \leqslant c \cdot\|f\|$ for all $f \in C[a, b]$,

(ii) $\left\|d\left(A_{n}(h, x)\right) / d x\right\| \leqslant c \cdot\left\|h^{\prime}\right\|$ for all $h \in C^{1}[a, b]$,

(iii) $\left|A_{n}(h, x)-h(x)\right| \leqslant c \cdot\left(\varepsilon_{n} \sqrt{(x-a)(b-x)}+\varepsilon_{n}^{2}\right) \cdot\left\|h^{\prime}\right\|$ for all $h \in C^{1}[a, b]$, all $a \leqslant x \leqslant b$ and $0 \leqslant \varepsilon_{n} \leqslant 2$.

Then for all $f \in C[a, b]$ we have

$$
\left|A_{n}^{+}(f, x)-f(x)\right| \leqslant c \cdot \omega\left(f, \varepsilon_{n} \sqrt{(x-a)(b-x)}\right) .
$$

Proof: Using the method applied by Lehnhoff in [15], among others, we distinguish three cases:

CASE (A). $\varepsilon_{n} \leqslant \sqrt{(x-a)(b-x)}, a \leqslant x \leqslant b$, which implies $\varepsilon_{n}^{2} \leqslant \varepsilon_{n} \sqrt{(x-a)(b-x)}$. 
If $h \in C^{1}[a, b]$, from (2.1) and condition (iii) we have

$$
\begin{aligned}
\mid h(x) & -A_{n}^{+}(h, x) \mid \\
& \leqslant\left|h(x)-A_{n}(h, x)\right|+\frac{x-a}{b-a}\left|h(b)-A_{n}(h, b)\right|+\frac{b-x}{b-a}\left|h(a)-A_{n}(h, a)\right| \\
& \leqslant c \cdot\left(\varepsilon_{n} \sqrt{(x-a)(b-x)}+\varepsilon_{n}^{2}\right) \cdot\left\|h^{\prime}\right\|+c \cdot \varepsilon_{n}^{2} \cdot\left\|h^{\prime}\right\|+c \cdot \varepsilon_{n}^{2} \cdot\left\|h^{\prime}\right\| \\
& \leqslant c \cdot\left(\varepsilon_{n} \sqrt{(x-a)(b-x)}+\varepsilon_{n}^{2}\right) \cdot\left\|h^{\prime}\right\| \\
& \leqslant c \cdot \varepsilon_{n} \sqrt{(x-a)(b-x)} \cdot\left\|h^{\prime}\right\| .
\end{aligned}
$$

CASE (B). $\sqrt{(x-a)(b-x)} \leqslant \varepsilon_{n},(a+b) / 2 \leqslant x \leqslant b$, hence $(x-a)(b-x) \leqslant$ $\epsilon_{n} \sqrt{(x-a)(b-x)}$. From (2.1) we get

$$
\begin{aligned}
h(x)-A_{n}^{+}(h, x)= & {[h(x)-h(b)]-\left[A_{n}(h, x)-A_{n}(h, b)\right] } \\
& +\frac{b-x}{b-a}\left\{\left[h(b)-A_{n}(h, b)\right]-\left[h(a)-A_{n}(h, a)\right]\right\} .
\end{aligned}
$$

Defining

$$
I_{n}(x):=\left|A_{n}(h, x)-A_{n}(h, b)\right| \text {, }
$$

we have from (iii)

$$
\begin{aligned}
\mid h(x) & -A_{n}^{+}(h, x) \mid \\
& \leqslant|h(x)-h(b)|+I_{n}(x)+\frac{b-x}{b-a}\left\{\left|h(b)-A_{n}(h, b)\right|+\left|h(a)-A_{n}(h, a)\right|\right\} \\
& \leqslant(b-x)\left\|h^{\prime}\right\|+I_{n}(x)+\frac{c(b-x)}{b-a} \cdot \varepsilon_{n}^{2} \cdot\left\|h^{\prime}\right\| .
\end{aligned}
$$

By condition (ii),

$$
\left\|\frac{d}{d v} A_{n}(h, v)\right\| \leqslant c \cdot\left\|h^{\prime}\right\|,
$$

and thus

$$
\begin{aligned}
I_{n}(x) & =\left|\int_{x}^{b}\left(\frac{d}{d v} A_{n}(h, v)\right) d v\right| \leqslant \int_{z}^{b}\left|\frac{d}{d v} A_{n}(h, v)\right| d v \\
& \leqslant c(b-x) \cdot\left\|h^{\prime}\right\| .
\end{aligned}
$$

This implies

$$
\begin{aligned}
\mid h(x) & -A_{n}^{+}(h, x) \mid \\
\leqslant & (b-x) \cdot\left\|h^{\prime}\right\|+c(b-x) \cdot\left\|h^{\prime}\right\|+4 \cdot \frac{c(b-x)}{b-a}\left\|h^{\prime}\right\| \\
\leqslant & c(b-x) \cdot\left\|h^{\prime}\right\| .
\end{aligned}
$$


Using the fact that

we get

$$
\begin{gathered}
1 \leqslant \frac{2(x-a)}{b-a} \text { for } \frac{a+b}{2} \leqslant x \leqslant b, \\
(b-x) \leqslant \frac{2(x-a)(b-x)}{b-a} .
\end{gathered}
$$

Thus

$$
\begin{aligned}
\left|h(x)-A_{n}^{+}(h, x)\right| & \leqslant \frac{2 c}{b-a}(x-a)(b-x) \cdot\left\|h^{\prime}\right\| \\
& \leqslant c \cdot \varepsilon_{n} \sqrt{(x-a)(b-x)} \cdot\left\|h^{\prime}\right\| .
\end{aligned}
$$

CASE (c). $\sqrt{(x-a)(b-x)} \leqslant \varepsilon_{n}, a \leqslant x \leqslant(a+b) / 2$, hence $(x-a)(b-x) \leqslant$ $\varepsilon_{n} \sqrt{(x-a)(b-x)}$. From (2.1) we get

$$
\begin{aligned}
h(x)-A_{n}^{+}(h, x)= & {[h(x)-h(a)]+\left[A_{n}(h, a)-A_{n}(h, x)\right] } \\
& +\frac{x-a}{b-a}\left\{\left[h(a)-A_{n}(h, a)\right]+\left[A_{n}(h, b)-h(b)\right]\right\} .
\end{aligned}
$$

On account of the fact that $x-a \leqslant 2(x-a)(b-x) /(b-a)$ for $a \leqslant x \leqslant(a+b) / 2$, we get again, by means of a method analogous to the one used in Case (B),

$$
\left|h(x)-A_{n}^{+}(h, x)\right| \leqslant c \cdot \varepsilon_{n} \sqrt{(x-a)(b-x)} \cdot\left\|h^{\prime}\right\| .
$$

Combining (2.2), (2.4), and (2.6) we have, for $n \in N$ and $a \leqslant x \leqslant b$,

$$
\left|h(x)-A_{n}^{+}(h, x)\right| \leqslant c \cdot \varepsilon_{n} \sqrt{(x-a)(b-x)} \cdot\left\|h^{\prime}\right\| .
$$

Since $\sqrt{(x-a)(b-x)} \leqslant(b-a) / 2(a \leqslant x \leqslant b)$ we obtain

$$
0 \leqslant \varepsilon_{n} \sqrt{(x-a)(b-x)} \leqslant b-a .
$$

From (2.1) and condition (i) we have

$$
\left\|A_{n}^{+} f\right\| \leqslant c \cdot\|f\| \text { for } f \in C[a, b] .
$$

Now, from (2.7) and (2.8) and using Lemma 1, we obtain Theorem 1.

The following is a generalisation of Theorem 5.6 in [3].

Theorem 2. Let $A_{n}: C[a, b] \rightarrow C^{1}[a, b]$ be a sequence of positive linear operators satisfying the following conditions:

(i) $A_{n}(1, x)=1, a \leqslant x \leqslant b$,

(ii) $A_{n}(|t-x|, x) \leqslant c \cdot\left(\varepsilon_{n} \sqrt{(x-a)(b-x)}+\varepsilon_{n}^{2}\right), a \leqslant x \leqslant b, 0 \leqslant \varepsilon_{n} \leqslant 2$,

(iii) $\left\|d A_{n}(h, x) / d x\right\| \leqslant c \cdot\left\|h^{\prime}\right\|$ for all $h \in C^{1}[a, b]$. 
Then for all $f \in C[a, b]$,

$$
\left|A_{n}^{+}(f, x)-f(x)\right| \leqslant c \cdot \omega\left(f, \varepsilon_{n} \sqrt{(x-a)(b-x)}\right) .
$$

Proof: Note that, for $f \in C[a, b]$,

$$
\left|A_{n}(f, x)\right| \leqslant\left|A_{n}(1, x)\right|\|f\|=\|f\|, \text { and thus }\left\|A_{n}(f)\right\| \leqslant\|f\| .
$$

Since $A_{n}$ is a sequence of positive linear operators and because of conditions (i) and (ii), we have for $h \in C^{1}[a, b]$

$$
\begin{aligned}
\left|A_{n}(h(t), x)-h(x)\right| & =\left|A_{n}(h(t)-h(x), x)\right| \\
& \leqslant A_{n}(|h(t)-h(x)|, x) \\
& \leqslant\left\|h^{\prime}\right\| \cdot A_{n}(|t-x|, x) \\
& \leqslant c \cdot\left(\varepsilon_{n} \sqrt{(x-a)(b-x)}+\varepsilon_{n}^{2}\right) \cdot\left\|h^{\prime}\right\| .
\end{aligned}
$$

From (2.9), (2.10), condition (iii) and Theorem 1 one arrives at the claim of Theorem 2.

\section{Application: Telyakovskin-type estimates}

In this section we shall demonstrate how estimates of the Telyakovskii-type can be derived from Timan-type inequalities. In the sequel, let

$$
\triangle_{n}(x):=\max \left\{\left(1-x^{2}\right)^{1 / 2} \cdot n^{-1}, n^{-2}\right\},|x| \leqslant 1 .
$$

We shall need the following auxiliary result from [3, Lemma 5.4].

LEMMA 2. Let $n \geqslant 1, m(n) \in \mathrm{N} \cup\{0\}$ and $c n \leqslant m(n) \leqslant \tilde{c} n(n \geqslant 2)$. If, for $p_{m(n)} \in \Pi_{m(n)}$ and $f \in C^{1}[-1,1]$,

$$
\left|f(x)-p_{m(n)}(x)\right| \leqslant c \cdot \Delta_{n}(x) \cdot\left\|f^{\prime}\right\|,
$$

then

$$
\left|f^{\prime}(x)-p_{m(n)}^{\prime}(x)\right| \leqslant c \cdot\left\|f^{\prime}\right\| \text {. }
$$

The main result of this note is contained in

THEOREM 3. Let $n \geqslant 1, m(n) \in N \cup\{0\}$ and $c n \leqslant m(n) \leqslant \tilde{c} n(n \geqslant 2)$. Let $A_{n}: C[-1,1] \rightarrow \Pi_{m(n)}$ be a sequence of linear operators. Suppose that, for all $f \in C[-1,1]$, the following Timan-type estimate holds:

$$
\left|A_{n}(f, x)-f(x)\right| \leqslant c \cdot \omega\left(f,\left(1-x^{2}\right)^{1 / 2} \cdot n^{-1}+n^{-2}\right),|x| \leqslant 1 .
$$


Then for $A_{n}^{+}$we have the Telyakovski-type inequality

$$
\left|A_{n}^{+}(f, x)-f(x)\right| \leqslant c \cdot \omega\left(f,\left(1-x^{2}\right)^{1 / 2} \cdot n^{-1}\right),|x| \leqslant 1 .
$$

Proof: Using elementary properties of the modulus of continuity we obtain from (3.1) for $f \in C[a, b]$

$$
\left|A_{\mathbf{n}}(f, x)-f(x)\right| \leqslant 2 c \cdot\|f\|,
$$

hence

$$
\left\|A_{n} f\right\| \leqslant c \cdot\|f\| .
$$

Furthermore, from (3.1) we have for $h \in C^{1}[a, b]$

$$
\begin{aligned}
\left|A_{n}(h, x)-h(x)\right| & \leqslant c\left(\left(1-x^{2}\right)^{1 / 2} \cdot n^{-1}+n^{-2}\right) \cdot\left\|h^{\prime}\right\| \\
& \leqslant 2 c \triangle_{n}(x) \cdot\left\|h^{\prime}\right\| .
\end{aligned}
$$

Using Lemma 2 we arrive at

$$
\left|\frac{d}{d x} A_{n}(h, x)-h^{\prime}(x)\right| \leqslant c \cdot\left\|h^{\prime}\right\|,
$$

and hence

$$
\left\|\frac{d}{d x} A_{n}(h, x)\right\| \leqslant c \cdot\left\|h^{\prime}\right\| .
$$

Combining (3.3), (3.4), (3.5) and Theorem 1 we obtain Theorem 3.

Corollary 1. (compare [3, Theorem 5.6]). Let $n \geqslant 1, m(n) \in N \cup\{0\}$, and $c n \leqslant m(n) \leqslant \tilde{c} n(n \geqslant 2)$. Furthermore, let $A_{n}: C[-1,1] \rightarrow \Pi_{m(n)}$ be a sequence of positive linear operators, satisfying the following conditions:

(i) $A_{n}(1, x)=1$,

(ii) $A_{n}(|t-x|, x)=O\left(\sqrt{1-x^{2}} \cdot n^{-1}+n^{-2}\right), n \rightarrow \infty$.

Then we have for $f \in C[-1,1]$

$$
\left|A_{n}^{+}(f, x)-f(x)\right| \leqslant c \cdot \omega\left(f, \sqrt{1-x^{2}} \cdot n^{-1}\right),|x| \leqslant 1 .
$$

Proof: Since $A_{n}$ is a sequence of positive linear operators and because of conditions (i) and (ii), we have for $f \in C[-1,1]$, using Popoviciu's theorem (see [22]), the pointwise inequality

$$
\begin{aligned}
\left|A_{n}(f, x)-f(x)\right| & \leqslant 2 \cdot \omega\left(f, A_{n}(|t-x|, x)\right) \\
& \leqslant c \cdot \omega\left(f, \sqrt{1-x^{2}} \cdot n^{-1}+n^{-2}\right),|x| \leqslant 1 .
\end{aligned}
$$

Using Theorem 3 we obtain Corollary 1.

For the Boolean sum modifications of the operators $G_{m(n)}$ introduced in Section 1 we have 
Corollary 2. (compare $[1,2])$. Let $n \geqslant 1, c n \leqslant m(n) \leqslant \tilde{c} n(n \geqslant 2)$ and $K_{m(n)}(v) \geqslant 0$. If $1-\rho_{1, m(n)}=\mathcal{O}\left(n^{-2}\right)$, then for $f \in C[-1,1]$ there holds the following

$$
\left|G_{m(n)}^{+}(f, x)-f(x)\right| \leqslant c \cdot \omega\left(f, \sqrt{1-x^{2}} \cdot n^{-1}\right),|x| \leqslant 1 .
$$

Proof: Since $1-\rho_{1, m(n)}=\mathcal{O}\left(n^{-2}\right)$ we have (see $\left.[1,2]\right)$

$$
\begin{aligned}
\mid f(x) & -G_{m(n)}(f, x) \mid \\
& \leqslant 2 \cdot \omega\left(f,\left(1-\rho_{1, m(n)}\right) \cdot|x|+\sqrt{2} \cdot \sqrt{1-\rho_{1, m(n)}} \cdot \sqrt{1-x^{2}}\right) \\
& =\mathcal{O}\left(\omega\left(f, \sqrt{1-x^{2}} \cdot n^{-1}+n^{-2}\right)\right) .
\end{aligned}
$$

Now Theorem 3 immediately yields the estimate for $G_{m(n)}^{+}$.

Note that it was shown by DeVore in $[6, p .81]$ that the relationship $1-\rho_{1, m(n)}=$ $\mathcal{O}\left(n^{-2}\right), n \rightarrow \infty$, holds true for the Matsuoka kernels $K_{\text {sn-s }}$, with $s \geqslant 2$.

REMARK 1. There are many further applications of Theorem 3. Today a large number of different proofs of the Timan theorem are known. As examples we mention the work of Freud and Vértesi [9], Freud and Sharma [8], Mills and Varma [19, 29], Saxena [23], Varma [28], Vértesi and Kis [30], Gonska and Cao [3] who constructed linear algebraic polynomial operators $W_{n}$ satisfying Timan-type estimates. By Theorem 3 , for the corresponding operators $W_{n}^{+}$it is clear that they give Telyakovskii-type estimates and thus provide a solution to Lorentz' problem for arbitrary continuous functions.

\section{FURTHER APPLICATIONS}

We give three applications of Theorem 2 for positive linear operators, all of which are related to the classical Bernstein operators.

EXAMPLE 1. Let $\alpha_{n} \geqslant 0$ and $f \in C[0,1]$. The Bernstein-Schurer polynomials are defined by (see [24])

$$
B_{n}\left(\alpha_{n}, f, x\right):=\sum_{i=0}^{n} f\left(\frac{i}{n+\alpha_{n}}\right) p_{n, i}(x), p_{n, i}(x):=\left(\begin{array}{c}
n \\
i
\end{array}\right) x^{i}(1-x)^{n-i} .
$$

If $\alpha_{n}=0$, then $B_{n}\left(\alpha_{n}, f, x\right)$ becomes the Bernstein polynomial $B_{n}(f, x)$.

LEMma 3 . The following equalities hold:

$$
B_{n}\left(\alpha_{n}, 1, x\right)=1, \quad B_{n}\left(\alpha_{n},(t-x)^{2}, x\right)=\frac{n x(1-x)+\alpha_{n}^{2} x^{2}}{\left(n+\alpha_{n}\right)^{2}}
$$


Proof: For a proof see Lemma 2.2 and Corollary 2.4 in [12].

Since $B_{n}\left(\alpha_{n}, f, 0\right)=f(0), B_{n}\left(\alpha_{n}, f, 1\right)=f\left(n /\left(n+\alpha_{n}\right)\right)$, we have

$$
\begin{aligned}
B_{n}^{+}\left(\alpha_{n}, f, x\right) & =B_{n}\left(\alpha_{n}, f, x\right)+x\left[f(1)-B_{n}\left(\alpha_{n}, f, 1\right)\right]+(1-x)\left[f(0)-B_{n}\left(\alpha_{n}, f, 0\right)\right] \\
& =B_{n}\left(\alpha_{n}, f, x\right)+x\left[f(1)-f\left(n /\left(n+\alpha_{n}\right)\right)\right]
\end{aligned}
$$

TheOREM 4. Let $f \in C[0,1], 0 \leqslant x \leqslant 1$, and $\beta_{n}:=\max \left(1 / \sqrt{n}, \sqrt{\alpha_{n}} / \sqrt{n}\right)$. If $S:=\left\{n \in N: \alpha_{n} \leqslant n\right\}$, then

$$
\left|B_{n}^{+}\left(\alpha_{n}, f, x\right)-f(x)\right| \leqslant c \cdot \omega\left(f, \beta_{n} \sqrt{x(1-x)}\right), \quad n \in S
$$

Proof: Note first that $B_{n}\left(\alpha_{n}, f, x\right)$ is a positive linear operator. Using Lemma 3 we have

$$
\begin{aligned}
B_{n}\left(\alpha_{n}, 1, x\right) & =1 \\
B_{n}\left(\alpha_{n},(t-x)^{2}, x\right) & \leqslant \frac{x(1-x)}{n}+\left(\frac{\alpha_{n}}{n}\right)^{2},
\end{aligned}
$$

hence (see $[\mathbf{1 3}]$ )

$$
\begin{aligned}
B_{n}\left(\alpha_{n},|t-x|, x\right) & \leqslant \sqrt{B_{n}\left(\alpha_{n},(t-x)^{2}, x\right) \cdot B_{n}\left(\alpha_{n}, 1, x\right)} \\
& \leqslant \sqrt{\frac{x(1-x)}{n}+\left(\frac{\alpha_{n}}{n}\right)^{2}} \leqslant \sqrt{\frac{x(1-x)}{n}}+\frac{\alpha_{n}}{n} .
\end{aligned}
$$

In view of the definition of $\beta_{n}$ we have

$$
\beta_{n} \geqslant \frac{1}{\sqrt{n}}, \beta_{n} \geqslant \frac{\sqrt{\alpha_{n}}}{\sqrt{n}} n \beta_{n}^{2} \geqslant \frac{\alpha_{n}}{n}
$$

and so

$$
B_{n}\left(\alpha_{n},|t-x|, x\right) \leqslant \beta_{n} \sqrt{x(1-x)}+\beta_{n}^{2}
$$

Note also that, for $n \in S$, we have

$$
\frac{\sqrt{\alpha_{n}}}{\sqrt{n}} \leqslant 1 \text { and } 0<\beta_{n} \leqslant 1
$$

Furthermore, from Lorentz [16] it is known that

$$
\frac{d}{d x} B_{n}(f, x)=n \cdot \sum_{i=0}^{n-1}\left\{f\left(\frac{i+1}{n}\right)-f\left(\frac{i}{n}\right)\right\} p_{n-1, i}(x), f \in C[0,1] .
$$


Similarly, if $h \in C^{1}[0,1]$, we get

$$
\begin{aligned}
\frac{d}{d x} B_{n}\left(\alpha_{n}, h, x\right) & =n \cdot \sum_{i=0}^{n-1}\left\{h\left(\frac{i+1}{n+\alpha_{n}}\right)-h\left(\frac{i}{n+\alpha_{n}}\right)\right\} p_{n-1, i}(x) \\
& =\frac{n}{n+\alpha_{n}} \sum_{i=0}^{n-1} h^{\prime}\left(\xi_{n, i}\right) p_{n-1, i}(x),
\end{aligned}
$$

hence

$$
\left\|\frac{d}{d x} B_{n}\left(\alpha_{n}, h, x\right)\right\| \leqslant \frac{n}{n+\alpha_{n}} \cdot\left\|h^{\prime}\right\| \leqslant\left\|h^{\prime}\right\| .
$$

Combining (4.1), (4.3), (4.4) and Theorem 2 we obtain the claim of Theorem 4.

COROLlaRY 3. Let $\lim _{n \rightarrow \infty} \alpha_{n} / n=0$. Then

$$
\lim _{n \rightarrow \infty}\left\|B_{n}^{+}\left(\alpha_{n}, f, x\right)-f(x)\right\|=0 .
$$

EXAMPLE 2. Let $f \in L[0,1]$ (the space of Lebesgue integrable functions defined on $[0,1]$ ), and $F_{1}(u):=\int_{0}^{u} f(t) d t$. Then the Kantorovič polynomials are defined by (see [16])

$$
\begin{aligned}
P_{n}(f(t), x) & :=\frac{d}{d x} B_{n+1}\left(F_{1}(u), x\right) \\
& =(n+1) \cdot \sum_{i=0}^{n}\left\{\int_{\frac{i}{n+1}}^{\frac{i+1}{n+1}} f(t) d t\right\} \cdot p_{n, i}(x) .
\end{aligned}
$$

We also define the function $F_{2}$ by $F_{2}(u):=\int_{0}^{u}\left(\int_{0}^{v} f(t) d t\right) d v$. Nagel investigated the Kantorovič operators of second order $Q_{n}$ given by (see [20])

$$
Q_{n}(f(t), x):=\frac{d}{d x} P_{n+1}\left(F_{1}(v), x\right)=\left(\frac{d}{d x}\right)^{2} B_{n+2}\left(F_{2}(u), x\right) .
$$

THEOREM 5. Let $f \in C[0,1]$ and $0 \leqslant x \leqslant 1$; then

$$
\left|P_{n}^{+}(f, x)-f(x)\right| \leqslant c \cdot \omega\left(f, \sqrt{\frac{x(1-x)}{n+1}}\right) .
$$

Proof: $P_{n}$ is a sequence of positive linear operators (see [18]), satisfying

$$
\begin{aligned}
P_{n}(1, x) & =1, \\
P_{n}\left((t-x)^{2}, x\right) & =\frac{n-1}{(n+1)^{2}} x(1-x)+\frac{1}{3(n+1)^{2}} \\
& \leqslant \frac{x(1-x)}{n+1}+\frac{1}{(n+1)^{2}},
\end{aligned}
$$


and

$$
\begin{aligned}
P_{n}(|t-x|, x) & \leqslant \sqrt{P_{n}\left((t-x)^{2}, x\right) \cdot P_{n}(1, x)} \\
& \leqslant \sqrt{\frac{x(1-x)}{n+1}}+\frac{1}{n+1}
\end{aligned}
$$

Furthermore, if $h \in C^{1}[0,1]$, then $d P_{n}(h, x) / d x=(d / d x)^{2} B_{n+1}\left(\int_{0}^{u} h(v) d v, x\right)$, where

$$
\begin{aligned}
\int_{0}^{u} h(v) d v & =\int_{0}^{u}\left(\int_{0}^{v} h^{\prime}(t) d t\right) d v+\int_{0}^{u} h(0) d v \\
& =\int_{0}^{u}\left(\int_{0}^{v} h^{\prime}(t) d t\right) d v+h(0) u
\end{aligned}
$$

From (4.5) we have

$$
\begin{aligned}
\frac{d}{d x} P_{n}(h, x) & =\left(\frac{d}{d x}\right)^{2} B_{n+1}\left[\int_{0}^{u}\left(\int_{0}^{v} h^{\prime}(t) d t\right) d v, x\right]+h(0)\left(\frac{d}{d x}\right)^{2} B_{n+1}(u, x) \\
& =\left(\frac{d}{d x}\right)^{2} B_{n+1}\left[\int_{0}^{u}\left(\int_{0}^{v} h^{\prime}(t) d t\right) d v, x\right] \\
& =Q_{n-1}\left(h^{\prime}, x\right) .
\end{aligned}
$$

Nagel [20] proved that the $Q_{n}$ are positive linear operators and that

$Q_{n}(1, x)=1-(n+2)^{-1}$.
Hence $\quad\left|Q_{n-1}\left(h^{\prime}, x\right)\right| \leqslant Q_{n-1}(1, x) \cdot\left\|h^{\prime}\right\| \leqslant\left\|h^{\prime}\right\|$,

and, consequently,

$$
\left\|\frac{d}{d x} P_{n}(h, x)\right\| \leqslant\left\|h^{\prime}\right\| .
$$

Combining (4.6), (4.7), (4.8), and using Theorem 2, we obtain Theorem 5.

EXAMPLE 3. For $f \in L[0,1]$, the so-called Durrmeyer operators [5] are given by

$$
M_{n}(f, x):=(n+1) \cdot \sum_{i=0}^{n}\left\{\int_{0}^{1} p_{n, i}(t) f(t) d t\right\} \cdot p_{n, i}(x) .
$$

For their modifications we have 
Theorem 6. Let $f \in C[0,1], 0 \leqslant x \leqslant 1$; then

$$
\left|M_{n}^{+}(f, x)-f(x)\right| \leqslant c \cdot \omega\left[f, \frac{\sqrt{x(1-x)}}{\sqrt{n+2}}\right] .
$$

Proof: It was shown in [5] that $M_{n}$ is a sequence of positive linear operators for which

(4.9)

$$
\begin{aligned}
M_{n}(1, x) & =1 \\
M_{n}\left((t-x)^{2}, x\right) & =(n+1) \cdot \frac{2 n x(1-x)-6 x(1-x)+2}{(n+1)(n+2)(n+3)} \\
& \leqslant \frac{2 n x(1-x)}{(n+2)(n+3)}+\frac{2}{(n+2)(n+3)} \leqslant 2\left(\frac{x(1-x)}{n+2}+\frac{1}{(n+2)^{2}}\right),
\end{aligned}
$$

and consequently,

$$
M_{n}(|t-x|, x) \leqslant \sqrt{M_{n}\left((t-x)^{2}, x\right) \cdot M_{n}(1, x)} \leqslant \sqrt{2} \cdot\left(\frac{\sqrt{x(1-x)}}{\sqrt{n+2}}+\frac{1}{n+2}\right) .
$$

If $h \in C^{1}[0,1]$, we also have from [5] that

$$
\begin{aligned}
\int_{0}^{1} p_{n, i}(t) d t & =(n+1)^{-1}, \quad i=0,1, \ldots, n, \\
\frac{d}{d x} M_{n}(h, x) & =n \cdot \sum_{i=0}^{n-1} p_{n-1, i}(x) \cdot \int_{0}^{1} h^{\prime}(t) p_{n+1, i+1}(t) d t, \\
\left\|\frac{d}{d x} M_{n}(h, x)\right\| & \leqslant\left\|h^{\prime}\right\| \cdot n \cdot \sum_{i=0}^{n-1} p_{n-1, i}(x) \cdot \int_{0}^{1} p_{n+1, i+1}(t) d t \\
& =\frac{n}{n+2} \cdot\left\|h^{\prime}\right\| \leqslant\left\|h^{\prime}\right\| .
\end{aligned}
$$

(4.9) through (4.11) and Theorem 2 now imply Theorem 6.

REMARK 2. The present authors proved in [4] that $\omega(f, \delta)$ in Theorems 5 and 6 may be replaced by $\omega_{2}(f, \delta)$, the second order modulus of continuity of $f$.

\section{REFERENCES}

[1] Jia-Ding Cao, 'Generalizations of Timan's theorem, Lehnhoff's theorem and Teljakowskiî's theorem (Chinese)', Kexue Tongbao 15 (1986), 1132-1135. 
[2] Jia-Ding Cao, 'Generalizations of Timan's theorem, Lehnhoff's theorem and Teljakowskiì's theorem', Schriftenreihe des Fachbereichs Mathematik SM-DU 106 (1986).

[3] Jia-Ding Cao and H.H. Gonska, 'Approximation by Boolean sums of positive linear operators II: Estimates for some numerical approximation schemes', Numer. Funct. Anal. Optim. 10 (1989), 643-672.

[4] Jia-Ding Cao and H.H. Gonska, 'Pointwise estimates for modified positive linear operators', Portugal. Math. 46 (1989), 401-430.

[5] M.M. Derrienic, 'Sur l'approximation des fonctions intégrables sur $[0,1]$ par des polynômes de Bernstein modifiés', J. Approx. Theory 31 (1981), 325-343.

[6] R.A. DeVore, The Approximation of Continuous Functions by Positive Linear Operators (Springer-Verlag, Berlin, Heidelberg, New York, 1972).

[7] R.A. DeVore, 'Degree of approximation', in Approximation Theory II: Proc. Int. Sympos. Austin, Editor G.G. Lorentz et al, pp. 117-161 (Academic Press, New York, 1976).

[8] G. Freud and A. Sharms, 'Some good sequences of interpolatory polynomials', Canad. J. Math. 26 (1974), 233-246.

[9] G. Freud and P. Vértesi, 'A new proof of A.F. Timan's approximation theorem', Studia Sci. Math. Hungar. 2 (1967), 403-414.

[10] H.H. Gonska, 'On Pičugov-Lehnhoff operators', Schriftenreihe des Fachbereichs Mathematik SM-DU 86 (1985).

[11] H.H. Gonska, 'Modified Pičugov-Lehnhoff operators', in Approximation Theory V: Proc. Int. Sympos. College Station, Editor C.K. Chui et al, pp. 355-358, 1986. (Acad. Press, New York).

[12] H.H. Gonska and J. Meier, 'Quantitative theorems on approximation by Bernstein-Stancu operators', Calcolo 21 (1984), 317-335.

[13] P.P. Korovkin, Linear Operators and Approximation Theory (Delhi, Hindustan, 1960).

[14] H.G. Lehnhoff, 'A simple proof of A.F. Timan's theorem', J. Approx. Theory 38 (1983), 172-176.

[15] H.G. Lehnhoff, 'A new proof of Teljakowskii's theorem', J. Approx. Theory 38 (1983), 177-181.

[16] G.G. Lorentz, Bernotein Polynomials (University of Toronto Press, Toronto, 1953).

[17] G.G. Lorentz, "Query in 'New and Unsolved Problems", in On Approximation Theory: Proc. Conference Oberwolfach, Editors P.L. Butzer and J. Korevaar, Pp. 179-199 (Birkhäuser, Basel, 1972 (2nd ed.)).

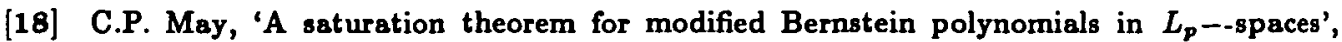
SIAM J. Math. Anal. 10 (1979), 321-330.

[19] T.M. Mills and A.K. Varma, 'A new proof of A.F. Timan's approximation theorem', Israel J. Math. 18 (1974), 39-44.

[20] J. Nagel, 'Kantorovič operators of second order', Monatsh. Math. 95 (1983), 33-44.

[21] S.A. Pičugov, 'Approximation of continuous functions on a segment by linear methods', (Russian), Mat. Zametki 24 (1978), 343-348.

[22] T. Popoviciu, 'Über die Konvergenz von Folgen positiver linearer Operatoren', An. Sttiinf. 
Univ. “Al. I. Cuza” Iagi Secf. I a Mat. (N.S.) 17 (1971), 123-132.

[23] R.B. Saxena, 'The approximation of continuous functions by interpolatory polynomials', Proc. Math. Inst. Bulgar. Akad. Sci. 12 (1970), 97-105.

[24] F. Schurer, 'On linear positive operators in approximation theory', Dissertation (Delft University of Technology, Delft, The Netherlands, 1965).

[25] S.A. Telyakovskir, 'Two theorems on the approximation of functions by algebraic polynomials', (Russian), Mat. Sb. (N.S.) 70 (1966), 252-265.

[28] A.F. Timan, 'Strengthening of Jackson's theorem on best approximation of continuous functions given on a finite interval of the real axis', (Russian), Dokl. Akad. Nauk SSSR 78 (1951), 17-20.

[27] A.F. Timan, Theory of Approximation of Functions of a Real Variable (Macmillan, New York, 1963).

[28] A.K. Varma, 'A new proof of A.F. Timan's approximation theorem II', J. Approx. Theory 18 (1976), 57-62.

[29] A.K. Varma and T.M. Mills, 'On the summability of Lagrange interpolation', J. Approx. Theory 9 (1973), 349-356.

[30] P. Vértesi and O. Kis, 'On a new interpolation process', Ann. Univ. Sci. Budapest 10 (1967), 117-128.

Department of Mathematics

Fudan University

Shanghai

Peoples Republic of China
Department of Computer Science

European Business School

D-6227 Oestrich-Winkel

West Germany 